\title{
UROTHELIAL CARCINOMA
}

\section{TARGETING PD-1 IN}

ADVANCED DISEASE

Data from the KEYNOTE-012

phase lb study demonstrate that pembrolizumab, an antibody targeting programmed cell death protein 1 (PD-1), has antitumour activity and an acceptable safety profile in patients with advanced urothelial carcinoma.

Several antibodies targeting PD-1 or its ligands are being studied for the treatment of urothelial cancer. Atezolizumab, which targets PD-L1, has been approved for use in patients whose disease progresses after platinum treatment. The phase lb KEYNOTE-012 study tests pembrolizumab in patients with advanced disease of various origins and includes one cohort with locally advanced or metastatic urothelial cancer. A total of 33 patients who had $\geq 1 \%$ PD-L1 expression in their tumour were enrolled in this cohort. Treatment consisted of $10 \mathrm{mg} / \mathrm{kg}$ intravenous pembrolizumab every 2 weeks for a maximum of 24 months.

The final analysis was performed on data from 27 patients. With a median treatment duration of 71 days (range 1-708 days) and a median follow-up period of 13 months (range 1-26 months), 26\% of patients had an overall response according to RECIST 1.1 (three patients had a complete response and four patients had a partial response; $P=0.0147$ ). In addition, four patients had stable disease. The median response duration was 10 months, three responses lasted $>12$ months and two complete responses were ongoing at the data cut-off point. Median overall survival was 13 months $(95 \% \mathrm{Cl}$ 5-20 months). Five patients and 15 patients had grade 3 or grade 1-2 treatment-related adverse events, respectively.

Clemens Thoma

ORIGINAL ARTICLE Plimack, E. R. et al. Safety and activity of pembrolizumab in patients with locally advanced or metastatic urothelial cancer (KEYNOTE-012): a non-randomised, open-label, phase $1 \mathrm{~b}$ study. Lancet Oncol. http://dx.doi. org/10.1016/S1470-2045(17)30007-4 (2017) 